$k$ ranges over the positive integers, are distinct, so $b$ has infinitely many distinct conjugates.

Thus (c) holds in $G$; it is well known that $G$ satisfies (b).

\title{
BIBLIOGRAPHY
}

1. R. H. Bruck, Difference sets in a finite group, Trans. Amer. Math. Soc. vol. 78 (1955) pp. 464-481.

2. M. Hall, Cyclic projective planes, Duke Math. J. vol. 14 (1947) pp. 1079-1090.

The University of Wisconsin

\section{MAXIMAL SUBALGEBRAS OF GROUP-ALGEBRAS}

JOHN WERMER

A closed subalgebra of a Banach algebra is called maximal if it is not contained in any larger proper closed subalgebra. Let $G$ be a discrete abelian topological group and $L$ its group-algebra, i.e. $L$ is the Banach algebra of functions $f$ on $G$ with $\sum_{\lambda \in G}|f(\lambda)|<\infty$ and multiplication defined as convolution. What are the maximal subalgebras of $L$ ? The complete answer is not known even when $G$ is the group of integers.

Here we assume that $G$ is ordered. Let $G^{+}$be the semi-group of nonnegative elements of $G$ and $L^{+}$the subset of $L$ consisting of functions which vanish outside of $G^{+}$. Then $L^{+}$is a proper closed subalgebra of $L$.

THEOREM $1 .^{1} L^{+}$is a maximal subalgebra of $L$ if and only if the ordering of $G$ is archimedean.

Proof. Suppose the ordering is non-archimedean. Then we can find $a, b$ in $G^{+}$with $n a<b$ for $n=1,2, \cdots$. Consider the set $G_{1}$ of all elements of $G$ of the form $g^{+}+n(-a)$, where $n=0,1,2, \cdots$ and $g^{+}$is in $G^{+}$. Clearly $G_{1}$ is a semi-group containing $G^{+}$and also $-a$ is in $G_{1}$ and $-b$ is not in $G_{1}$. Let $L_{1}$ be the closed subalgebra of $L$ consisting of all functions vanishing outside $G_{1}$. Then $L_{1}$ lies properly between $L^{+}$and $L$, whence $L^{+}$is not maximal.

Suppose now that the ordering of $G$ is archimedean. Let $\mathfrak{A}^{\prime}$ be a proper closed subalgebra of $L$ with $L^{+}$included in $\mathfrak{A}^{\prime}$. We shall show $\mathfrak{A}^{\prime}=L^{+}$.

Let $E_{\lambda}$ be the function in $L$ with $E_{\lambda}(g)=0, g \neq \lambda, E_{\lambda}(\lambda)=1$. Then

Received by the editors December 27, 1954.

${ }^{1}$ A proof of this theorem has also been found by I. M. Singer. See the note below. 
for some $\lambda_{0}>0, E_{-\lambda_{0}}$ is not in $\mathfrak{A}^{\prime}$. For $\mathfrak{A}^{\prime}$ contains $L^{+}$and so $E_{\lambda}$ is in $\mathfrak{A}^{\prime}$, for $\lambda>0$. If also all $E_{\lambda}$ with $\lambda<0$ were to belong to $\mathfrak{U}^{\prime}$, then $\mathfrak{A}^{\prime}$ should equal $L$. Thus for some $\lambda_{0}>0, E_{\lambda_{0}}$ is in $\mathfrak{A}^{\prime}$ and its inverse $E_{-\lambda_{0}}$ is not. By a basic result of Gelfand there hence exists a (linear) multiplicative functional $\chi$ on $\mathfrak{A}^{\prime}$ with $\chi\left(E_{\lambda_{0}}\right)=0$.

Consider any $\lambda_{1}>0$. Then since the ordering is archimedean, there is a positive integer $k$ with $-\lambda_{0}+k \lambda_{1}>0$. Hence $E_{-\lambda_{0}+k \lambda_{1}}$ is in $\mathfrak{I}^{\prime}$. We therefore get

$$
\left(\chi\left(E_{\lambda_{1}}\right)\right)^{k}=\chi\left(E_{k \lambda_{1}}\right)=\chi\left(E_{\lambda_{0}}\right) \chi\left(E_{-\lambda_{0}+k \lambda_{1}}\right)=0
$$

and so $\chi\left(E_{\lambda_{1}}\right)=0$ for all $\lambda_{1}>0$.

$L$ admits the involution

$$
f \rightarrow f^{*} \text { where } f^{*}(\lambda)=\overline{f(-\lambda)} .
$$

Let $\mathfrak{A}^{\prime \prime}$ be the closed subalgebra of $\mathfrak{A}^{\prime}$ generated by the self-adjoint elements in $\mathfrak{U}^{\prime}$. Then $\mathfrak{A}^{\prime \prime}$ is a self-adjoint subalgebra of $\mathfrak{A}^{\prime}$ and contains the unit element. The functional $\chi$ restricted to $\mathfrak{A}^{\prime \prime}$ defines a multiplicative functional on $\mathfrak{A}^{\prime \prime}$. Now a theorem due to Silov [1] asserts that a multiplicative functional defined on a closed selfadjoint subalgebra of a Banach algebra may be extended to a multiplicative functional defined on the whole Banach algebra. We apply this theorem to extend $\chi$ from $\mathfrak{U}^{\prime \prime}$ to a multiplicative functional $\chi_{0}$ defined on all of $L$.

We claim that if an element $f$ of $\mathfrak{A}^{\prime}$ has the form $f=\sum_{\lambda<0} a_{\lambda} E_{\lambda}$, then $\chi_{0}(f)=0$. For $f^{*}=\sum_{\lambda<0} \bar{a}_{\lambda} E_{-\lambda}$ is in $L^{+}$and so is in $\mathfrak{A}^{\prime}$. Hence $f$ and $f^{*}$ are in $\mathfrak{A}^{\prime}$, whence $f$ and $f^{*}$ are in $\mathfrak{I}^{\prime \prime}$. Then

But

$$
\chi_{0}\left(f^{*}\right)=\chi\left(f^{*}\right)=\sum_{\lambda<0} \bar{a}_{\lambda} \chi\left(E_{-\lambda}\right)=0 .
$$

$$
\chi_{0}(f)=\overline{\chi_{0}\left(f^{*}\right)},
$$

whence $\chi_{0}(f)=0$.

Take now any $\phi$ in $\mathfrak{A}^{\prime}$. Then $\phi=\sum_{\lambda \in G} \phi_{\lambda} E_{\lambda}, \sum\left|\phi_{\lambda}\right|<\infty$. Since each $E_{\lambda}$ with $\lambda>0$ is in $\mathfrak{H}^{\prime}, \sum_{\lambda<0} \phi_{\lambda} E_{\lambda}$ is in $\mathfrak{A}^{\prime}$. Fix $\lambda_{0}>0$. Then

$$
\left(\sum_{\lambda<0} \phi_{\lambda} E_{\lambda}\right) E_{\lambda_{0}}=\sum_{\lambda<-\lambda_{0}} \phi_{\lambda} E_{\lambda+\lambda_{0}}+\sum_{\lambda \geqq-\lambda_{0}} \phi_{\lambda} E_{\lambda+\lambda_{0}} .
$$

The left-hand term and the second term on the right are in $\mathfrak{A}^{\prime}$. Hence $\sum_{\lambda<-\lambda_{0}} \phi_{\lambda} E_{\lambda+\lambda_{0}}$ is in $\mathfrak{I}^{\prime}$. By the preceding, then,

$$
0=\chi_{0}\left(\sum_{\lambda<-\lambda_{0}} \phi_{\lambda} E_{\lambda+\lambda_{0}}\right)=\chi_{0}\left(\sum_{\lambda<-\lambda_{0}} \phi_{\lambda} E_{\lambda}\right) \cdot \chi_{0}\left(E_{\lambda_{0}}\right) \text {. }
$$


Hence $\chi_{0}\left(\sum_{\lambda<-\lambda_{0}} \phi_{\lambda} E_{\lambda}\right)=0$, since $\left|\chi_{0}\left(E_{\lambda_{0}}\right)\right|=1$. Choose $\lambda_{1}, 0 \leqq \lambda_{1}<\lambda_{0}$. We then have $\chi_{0}\left(\sum_{\lambda<-\lambda_{1}} \phi_{\lambda} E_{\lambda}\right)=0$. Hence $\chi_{0}\left(\sum_{-\lambda_{0} \leqq \lambda<-\lambda_{1}} \phi_{\lambda} E_{\lambda}\right)=0$ and so $\left|\chi_{0}\left(\phi_{-\lambda_{0}} E_{-\lambda_{0}}\right)\right|=\left|\chi_{0}\left(\sum_{-\lambda_{0}<\lambda<-\lambda_{1}} \phi_{\lambda} E_{\lambda}\right)\right| \leqq|| \sum_{-\lambda_{0}<\lambda<-\lambda_{1}} \phi_{\lambda} E_{\lambda}||$. Since $\sum\left|\phi_{\lambda}\right|<\infty$, we can choose $\lambda_{1}$ so that $\left\|\sum_{-\lambda_{0}<\lambda<-\lambda_{1}} \phi_{\lambda} E_{\lambda}\right\|$ $=\sum_{-\lambda_{0}<\lambda<-\lambda_{1}}\left|\phi_{\lambda}\right|<\epsilon$, for any given positive $\epsilon$. Then $\left|\phi_{-\lambda_{0}}\right|=$ $\left|\chi_{0}\left(\phi_{-\lambda_{0}} E_{-\lambda_{0}}\right)\right|<\epsilon$. Hence $\phi_{-\lambda_{0}}=0$. Since this holds for all $\lambda_{0}>0$, we conclude that $\phi$ is in $L^{+}$. Hence $\mathfrak{A}^{\prime}=L^{+}$, as asserted.

Note. I had first proved Theorem 1 for the group $G$ of integers (Bull. Amer. Math. Soc. Abstract 60-2-281), but did not publish the proof. After hearing from R. Arens and I. M. Singer about their work on archimedean ordered groups [2] I tried to extend my result to that situation and proved Theorem 1 given in this paper.

\section{REFERENCES}

1. G. W. Mackey, Commutative Banach algebras, Lecture notes (multigraphed), Harvard University, 1952, ed. by A. Blair, p. 95.

2. R. Arens and I. M. Singer, to appear.

BROWN UNIVERSITY 